\title{
Fuzzy Shortest Path Problem Based on Level $\lambda$-Triangular LR Fuzzy Numbers
}

\author{
S. Elizabeth and L. Sujatha \\ Department of Mathematics, Auxilium College (Autonomous), Tamil Nadu, Vellore 632006, India \\ Correspondence should be addressed to L. Sujatha, sujathajayasankar@yahoo.co.in
}

Received 15 April 2011; Accepted 1 October 2011

Academic Editor: Uzay Kaymak

Copyright ( $) 2012$ S. Elizabeth and L. Sujatha. This is an open access article distributed under the Creative Commons Attribution License, which permits unrestricted use, distribution, and reproduction in any medium, provided the original work is properly cited.

In problems of graphs involving uncertainties, the fuzzy shortest path problem is one of the most studied topics, since it has a wide range of applications in different areas and therefore deserves special attention. In this paper, algorithms are proposed for the fuzzy shortest path problem, where the arc length of the network takes imprecise numbers, instead of real numbers, namely, level $\lambda$-triangular LR fuzzy numbers. Few indices defined in this paper help to identify the shortest path in fuzzy environment.

\section{Introduction}

Many researchers have focused on fuzzy shortest path problem in a network, since it is important to many applications such as communications, routing, and transportation. In traditional shortest path problems, the arc length of the network takes precise numbers, but in the real-world problem, the arc length may represent transportation time or cost which can be known only approximately due to vagueness of information, and hence it can be considered a fuzzy number. The fuzzy set theory, proposed by Zadeh [1], is utilized to deal with uncertainty problems.

The fuzzy shortest path problem was first analysed by Dubois and Prade [2]. According to their approach, the shortest path length can be obtained, but the corresponding path in the network may not exist. Klein [3] proposed a dynamic programming recursion-based fuzzy algorithm. Lin and Chern [4] found the fuzzy shortest path length in a network by means of a fuzzy linear programming approach. Okada and Soper [5] proposed a fuzzy algorithm, which was based on multiple-labelling methods to offer nondominated paths to a decision maker. Chuang and Kung [6] proposed a fuzzy shortest path length procedure that can find a fuzzy shortest path length among all possible paths in a network. Yao and Lin [7] presented two new types of fuzzy shortest path network problems. The main results obtained from their studies were that the shortest path in the fuzzy sense corresponds to the actual paths in the network, and the fuzzy shortest path problem is an extension of the crisp case. Nayeem and Pal [8] have proposed an algorithm based on the acceptability index introduced by Sengupta and Pal [9] which gives a single fuzzy shortest path or a guideline for choosing the best fuzzy shortest path according to the decision maker's viewpoint. Thus, numerous papers have been published on the fuzzy shortest path problem (FSPP).

This paper is organized as follows. In Section 2, some elementary concepts and operations of fuzzy set theory have been reviewed. Also new indices have been defined for level $\lambda$-triangular LR fuzzy numbers. In Section 3, new algorithms have been proposed for fuzzy shortest path problem based on level $\lambda$-triangular LR indices. Finally, the paper is concluded in Section 4.

\section{Prerequisites}

Definition 1 (Acyclic digraph). A digraph is a graph each of whose edges are directed. Hence, an acyclic digraph is a directed graph without cycle.

Definition 2 (Level $\lambda$-triangular LR fuzzy number). Level $\lambda$-triangular LR fuzzy number is shown in Figure 1 and it 


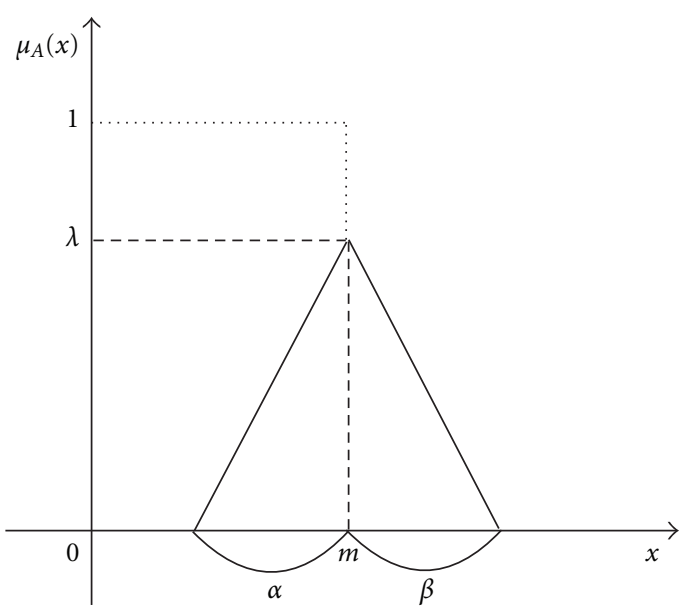

FIgURE 1: Level $\lambda$-triangular LR fuzzy number $A$.

is represented by $A=(m, \alpha, \beta ; \lambda)_{\mathrm{LR}}$ with the membership function

$$
\mu_{A}(x)= \begin{cases}0, & x \leq m-\alpha, \\ \frac{\lambda[x-(m-\alpha)]}{\alpha}, & m-\alpha \leq x \leq m, \\ \lambda, & x=m, \\ \frac{\lambda[(m+\beta)-x]}{\beta}, & m \leq x \leq m+\beta, \\ 0, & x \geq m+\beta .\end{cases}
$$

Here, $m$ is the point whose membership value is $\lambda$, where $0<\lambda \leq 1$ and $\alpha, \beta$ are the left hand and right hand spreads, respectively.

In addition, let $(m, \alpha, \beta ; 1)_{\mathrm{LR}}$ be the triangular LR fuzzy number, and it is denoted by $(m, \alpha, \beta)_{\mathrm{LR}}$.

Definition 3 (Addition operation on level $\lambda$-triangular LR fuzzy numbers). Let $L_{1}=\left(m_{1}, \alpha_{1}, \beta_{1} ; \lambda\right)_{\mathrm{LR}}$ and $L_{2}=$ $\left(m_{2}, \alpha_{2}, \beta_{2} ; \lambda\right)_{\mathrm{LR}}$ be the two level $\lambda$-triangular LR fuzzy numbers, then

$$
\begin{aligned}
L_{1}+L_{2} & =\left(m_{1}, \alpha_{1}, \beta_{1} ; \lambda\right)_{\mathrm{LR}}+\left(m_{2}, \alpha_{2}, \beta_{2} ; \lambda\right)_{\mathrm{LR}} \\
& =\left(m_{1}+m_{2}, \alpha_{1}+\alpha_{2}, \beta_{1}+\beta_{2} ; \lambda\right)_{\mathrm{LR}} .
\end{aligned}
$$

Definition 4 (Minimum operation on level $\lambda$-triangular LR fuzzy numbers [10]). Let $L_{1}=\left(m_{1}, \alpha_{1}, \beta_{1} ; \lambda\right)_{\mathrm{LR}}$ and $L_{2}=\left(m_{2}, \alpha_{2}, \beta_{2} ; \lambda\right)_{\mathrm{LR}}$ be the two level $\lambda$-triangular LR fuzzy numbers, then

$$
\begin{aligned}
L_{\min } & =\min \left(L_{1}, L_{2}\right) \\
& =\left(\min \left(m_{1}, m_{2}\right), \max \left(\alpha_{1}, \alpha_{2}\right), \min \left(\beta_{1}, \beta_{2}\right) ; \lambda\right)_{\mathrm{LR}} .
\end{aligned}
$$

In this paper, we introduce the following definitions.

Definition 5 (Level $\lambda$-triangular LR intersection index). Let the level $\lambda$-triangular LR ith fuzzy path length be $L_{i}=$ $\left(m_{i}, \alpha_{i}, \beta_{i} ; \lambda\right)_{\mathrm{LR}}$, and let the level $\lambda$-triangular LR fuzzy shortest length be $L_{\min }=(m, \alpha, \beta ; \lambda)_{\mathrm{LR}}, 0<\lambda \leq 1$ where $m-\alpha \leq m_{i}-\alpha_{i}, m \leq m_{i}, m+\beta \leq m_{i}+\beta_{i}$, then the

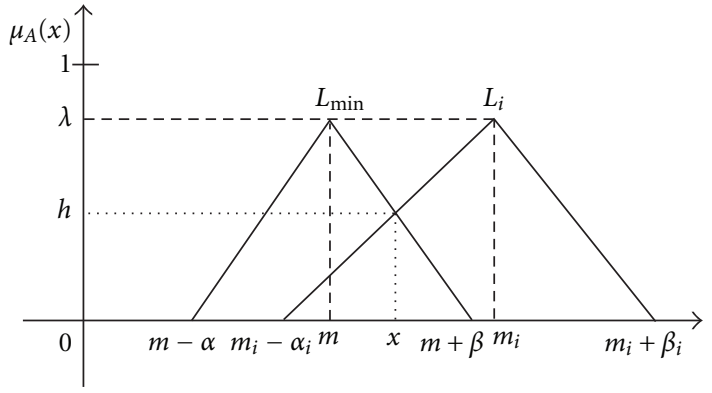

Figure 2: Level $\lambda$-triangular LR intersection index.

level $\lambda$-triangular LR intersection index between $L_{\min }$ and $L_{i}$ is calculated as

$$
T_{\mathrm{LRI}}\left(L_{\mathrm{min}}, L_{i}\right)=\frac{\lambda\left[(m+\beta)-\left(m_{i}-\alpha_{i}\right)\right]}{\alpha_{i}+\beta}=h .
$$

The above formula is obtained as follows.

Consider Figure 2:

Let $y_{d}$ be the membership function.

For $m \leq x \leq m+\beta$,

$$
\begin{gathered}
y_{d}=\frac{\lambda[(m+\beta)-x]}{(m+\beta)-m} \Longrightarrow \beta y_{d}=\lambda(m+\beta)-\lambda x, \\
\lambda x=\lambda(m+\beta)-\beta y_{d}, \\
x=\frac{\lambda(m+\beta)-\beta y_{d}}{\lambda} .
\end{gathered}
$$

For $m_{i}-\alpha_{i} \leq x \leq m_{i}$

$$
\begin{gathered}
y_{d}=\frac{\lambda\left[x-\left(m_{i}-\alpha_{i}\right)\right]}{m_{i}-m_{i}+\alpha_{i}} \Longrightarrow \alpha_{i} y_{d}=\lambda x-\lambda\left(m_{i}-\alpha_{i}\right), \\
\lambda x=\alpha_{i} y_{d}+\lambda\left(m_{i}-\alpha_{i}\right), \\
x=\frac{\alpha_{i} y_{d}+\lambda\left(m_{i}-\alpha_{i}\right)}{\lambda} .
\end{gathered}
$$

Equating (5) and (6),

$$
\begin{gathered}
\frac{\lambda(m+\beta)-\beta y_{d}}{\lambda}=\frac{\alpha_{i} y_{d}+\lambda\left(m_{i}-\alpha_{i}\right)}{\lambda}, \\
\lambda\left[(m+\beta)-\left(m_{i}-\alpha_{i}\right)\right]=\left(\alpha_{i}+\beta\right) y_{d}, \\
T_{\mathrm{LRI}}\left(L_{\mathrm{min}}, L_{i}\right)=y_{d}=\frac{\lambda\left[(m+\beta)-\left(m_{i}-\alpha_{i}\right)\right]}{\alpha_{i}+\beta}=h .
\end{gathered}
$$

In the level $\lambda$-triangular LR intersection index, we have $L_{1}<$ $L_{2}$ if and only if $T_{\mathrm{LRI}}\left(L_{\min }, L_{1}\right)>T_{\mathrm{LRI}}\left(L_{\min }, L_{2}\right)$.

Definition 6 (Indices based on $L_{\min }$ and $L_{i}$ ). Let the level $\lambda$ triangular LR $i$ th fuzzy path length be $L_{i}=\left(m_{i}, \alpha_{i}, \beta_{i} ; \lambda\right)_{\mathrm{LR}}$, 
and let the level $\lambda$-triangular LR fuzzy shortest length be $L_{\min }=(m, \alpha, \beta ; \lambda)_{\mathrm{LR}}, 0<\lambda \leq 1$ where $m-\alpha \leq m_{i}-\alpha_{i}$, $m \leq m_{i}, m+\beta \leq m_{i}+\beta_{i}$, then

(a) the level $\lambda$-triangular LR weighted average index between $L_{\min }$ and $L_{i}$ is calculated as

$Z_{i}^{*}\left(L_{\min }, L_{i}\right)=\frac{\lambda(m)+\lambda\left(m_{i}\right)}{\lambda+\lambda}=\frac{\lambda(m)+\lambda\left(m_{i}\right)}{2 \lambda}$.

Here, we have $L_{1}<L_{2}$ if and only if $Z_{1}^{*}\left(L_{\min }, L_{1}\right)<$ $Z_{2}^{*}\left(L_{\min }, L_{2}\right)$,

(b) the level $\lambda$-triangular LR Minkowski distance index between $L_{\min }$ and $L_{i}$ is calculated as

$$
d\left(L_{\min }, L_{i}\right)=\sqrt[w]{\mathfrak{f}+\left|(m+\beta)-\left(m_{i}+\beta_{i}\right)\right|^{w}}
$$

where $\mathfrak{f}$ denotes $\left|(m-\alpha)-\left(m_{i}-\alpha_{i}\right)\right|^{w}+\left|m-m_{i}\right|^{w}$ and $w \in[1, \infty]$. Here, we have $L_{1}<L_{2}$ if and only if $d\left(L_{\min }, L_{1}\right)<d\left(L_{\min }, L_{2}\right)$.

Generalizing Hamming distance and Euclidean distance results in Minkowski distance. It becomes the Hamming distance (HD) for $w=1$, while the Euclidean distance (ED) for $w=2$.
Definition 7 (Indices based on $L_{i}$ ). Let the level $\lambda$-triangular LR $i$ th fuzzy path length be $L_{i}=\left(m_{i}, \alpha_{i}, \beta_{i} ; \lambda\right)_{\mathrm{LR}}$, then

(a) the level $\lambda$-triangular LR mean index for $L_{i}$ is calculated as

$$
T_{\text {Mean }}\left(L_{i}\right)=\left[m_{i}+\left(\frac{\beta_{i}-\alpha_{i}}{2}\right)\right] .
$$

It is obtained as follows:

$$
T_{\text {Mean }}\left(L_{i}\right)=\frac{\int_{m_{i}-\alpha_{i}}^{m_{i}+\beta_{i}} \lambda x d x}{\int_{m_{i}-\alpha_{i}}^{m_{i}+\beta_{i}} \lambda d x}=\frac{2 m_{i}+\beta_{i}-\alpha_{i}}{2}
$$

(b) the level $\lambda$-triangular LR centroid index for $L_{i}$ is calculated as

$$
T_{\text {Centroid }}\left(L_{i}\right)=\left[m_{i}+\left(\frac{\beta_{i}-\alpha_{i}}{3}\right)\right]
$$

It is obtained as follows:

$$
T_{\text {Centroid }}\left(L_{i}\right)=\frac{\int_{m_{i}-\alpha_{i}}^{m_{i}}\left[\lambda\left[x-\left(m_{i}-\alpha_{i}\right)\right] / \alpha_{i}\right] x d x+\int_{m_{i}}^{m_{i}+\beta_{i}}\left[\lambda\left[\left(m_{i}+\beta_{i}\right)-x\right] / \beta_{i}\right] x d x}{\int_{m_{i}-\alpha_{i}}^{m_{i}}\left[\lambda\left[x-\left(m_{i}-\alpha_{i}\right)\right] / \alpha_{i}\right] d x+\int_{m_{i}}^{m_{i}+\beta_{i}}\left[\lambda\left[\left(m_{i}+\beta_{i}\right)-x\right] / \beta_{i}\right] d x}=\frac{3 m_{i}+\beta_{i}-\alpha_{i}}{3} .
$$

Crisp Graph with Fuzzy Weights (Type V) (Blue et al. [11]). A fifth type of graph fuzziness occurs when the graph has known vertices and edges, but unknown weights (or capacities) on the edges. Thus, only the weights are fuzzy.

Applications. To plan the quickest automobile route from one city to another. Unfortunately, the map gives distances, not travel times, so it is not known exactly how long it takes to travel any particular road segment.

Definition 8 (The signed distance [7]). $d(b, 0)=b$ when $b, 0 \in \mathbb{R}$, where $d(b, 0)$ means the signed distance of $b$ measuring from 0 .

Definition 9 (Signed distance of level $\lambda$-triangular fuzzy number $A$ [7]). For each $\lambda \in(0,1]$ and $A=(a, b, c ; \lambda)_{\mathrm{LR}}$, the signed distance from 0 to $A$ is defined by

$$
d(A, 0)=\frac{1}{4}(2 b+a+c)
$$

The ranking of level $\lambda$-triangular fuzzy numbers is defined by $A<B$ if and only if $d(A, 0)<d(B, 0)$.

For the sake of verification, Definition 9 is rewritten as the signed distance from 0 to $L_{1}=\left(m_{1}, \alpha_{1}, \beta_{1} ; \lambda\right)_{\mathrm{LR}}$, and it is defined by

$$
d\left(L_{1}, 0\right)=\frac{1}{4}\left[2 m_{1}+\left(m_{1}-\alpha_{1}\right)+\left(m_{1}+\beta_{1}\right)\right] .
$$

Here, $L_{1}<L_{2}$ if and only if $d\left(L_{1}, 0\right)<d\left(L_{2}, 0\right)$.

Definition 10 (Nayeem and Pal acceptability index [8]). Nayeem and Pal extended the acceptability index originally proposed by Sengupta and Pal [9] for interval numbers to triangular LR fuzzy numbers as follows.

If $a=\left(m_{1}, \alpha_{1}, \beta_{1}\right)_{\mathrm{LR}}$ and $b=\left(m_{2}, \alpha_{2}, \beta_{2}\right)_{\mathrm{LR}}$ are two triangular LR fuzzy numbers with $m_{1}-\alpha_{1} \leq m_{2}-\alpha_{2}$, $m_{1} \leq m_{2}, m_{1}+\beta_{1} \leq m_{2}+\beta_{2}$, then the acceptability index of the proposition " $a$ " preferred to " $b$ " is given by $A(a<$ $b)=\left(m_{2}-m_{1}\right) /\left(\beta_{1}+\alpha_{2}\right)$. Here, $a<b$ if and only if $A(a<b)>A(b<a)$.

To find the fuzzy shortest path, the above acceptability index was slightly modified in [10] as follows:

$$
A\left(L_{\min }<L_{i}\right)=\frac{m_{i}-m}{\beta+\alpha_{i}},
$$

where $L_{i}=\left(m_{i}, \alpha_{i}, \beta_{i}\right)_{\mathrm{LR}}$ is the triangular LR $i$ th fuzzy path length, and $L_{\min }=(m, \alpha, \beta)_{\mathrm{LR}}$ is the triangular LR fuzzy shortest length. Here, $L_{1}<L_{2}$ if and only if $A\left(L_{\min }<L_{1}\right)<$ $A\left(L_{\min }<L_{2}\right)$.

The acceptability index defined in [10] was obtained as follows. 


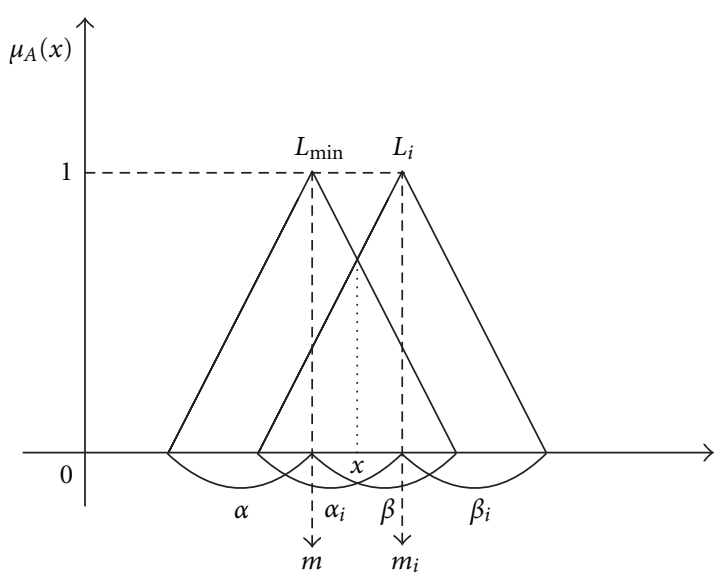

FIgURE 3: Triangular LR acceptability index.

Definition 11 (Triangular LR fuzzy number $A$ [12]). A fuzzy number $A$ is of LR type if there exists a reference function $L$ (for left) and $R$ (for right) and scalar $\alpha>0, \beta>0$ with

$$
\mu_{A}(x)= \begin{cases}L\left[\left(\frac{m-x}{\alpha}\right)\right], & x<m, \\ 1, & x=m, \\ R\left[\left(\frac{x-m}{\beta}\right)\right], & x>m,\end{cases}
$$

where " $m$ " is a real number whose membership value is 1 , and $\alpha, \beta$ are called the left and right spreads, respectively. Symbolically, $A$ is represented by $A=(m, \alpha, \beta)_{\mathrm{LR}}$.

Consider Figure 3: if $x<m_{i}$,

$$
y_{d}=\frac{m_{i}-x}{\alpha_{i}} \Longrightarrow x=m_{i}-\alpha_{i} y_{d}
$$

If $x>m$,

$$
y_{d}=\frac{x-m}{\beta} \Longrightarrow x=\beta y_{d}+m
$$

Equating (18) and (19),

$$
\begin{gathered}
m_{i}-\alpha_{i} y_{d}=\beta y_{d}+m, \\
m_{i}-m=\left(\beta+\alpha_{i}\right) y_{d}, \\
y_{d}=\frac{m_{i}-m}{\beta+\alpha_{i}} .
\end{gathered}
$$

This acceptability index can also be defined for level $\lambda$ triangular LR fuzzy numbers as follows:

$$
A\left(L_{\min }<L_{i}\right)=\frac{\lambda\left(m_{i}-m\right)}{\beta+\alpha_{i}},
$$

where $L_{i}=\left(m_{i}, \alpha_{i}, \beta_{i} ; \lambda\right)_{\mathrm{LR}}$ and $L_{\min }=(m, \alpha, \beta ; \lambda)_{\mathrm{LR}}$, for the sake of verification. If $\lambda=1$, we obtain the acceptability index defined in [10].

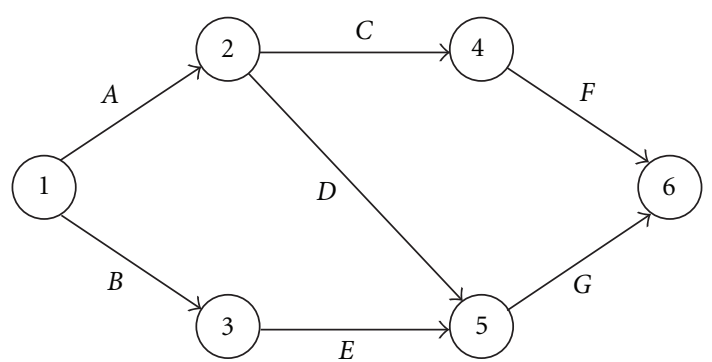

FIgURE 4: The classical network.

\section{Proposed Algorithm for Fuzzy Shortest Path Problem Based on Level $\lambda$-Triangular LR Fuzzy Numbers}

In many practical situations, we often need to employ a measurement tool to distinguish between two similar sets or groups. For this purpose, several similarity measures had been presented in [13-19].

In this paper, we introduce a new method called intersection index which acts as the measurement tool between $L_{\text {min }}$ and $L_{i}$. The larger the height of the intersection area of two triangles, the higher the intersection index will be between them. If there is no intersection area between $L_{\min }$ and $L_{i}$, then the intersection index is treated as zero. As mentioned previously, the similarity measure, namely, intersection index defined in Definition 5 will help the decision makers to decide which path is the shortest one. Using this concept, we now propose Algorithm 1 for FSPP.

\section{Example 12.}

Step 1. Construct a network with 6 vertices and 7 edges as cited in Figure 4.

let $\lambda=0.7$.

Assume the arc lengths as

$$
\begin{aligned}
& A(1-2)=(30,2,4 ; 0.7)_{\mathrm{LR}}, B(1-3)=(20,5,7 ; 0.7)_{\mathrm{LR}}, \\
& C(2-4)=(25,2,2 ; 0.7)_{\mathrm{LR}}, D(2-5)=(15,4,2 ; 0.7)_{\mathrm{LR}}, \\
& E(3-5)=(21,4,6 ; 0.7)_{\mathrm{LR}}, F(4-6)=(14,1,4 ; 0.7)_{\mathrm{LR}}, \\
& G(5-6)=(17,4,2 ; 0.7)_{\mathrm{LR}} .
\end{aligned}
$$

Step 2. The possible paths and the corresponding path lengths are as follows:

$$
\begin{aligned}
& P_{1}: 1-2-4-6 \text { with } A+C+F=(69,5,10 ; 0.7)_{\mathrm{LR}}=L_{1}, \\
& P_{2}: 1-2-5-6 \text { with } A+D+G=(62,10,8 ; 0.7)_{\mathrm{LR}}=L_{2}, \\
& P_{3}: 1-3-5-6 \text { with } B+E+G=(58,13,15 ; 0.7)_{\mathrm{LR}}=L_{3} .
\end{aligned}
$$

Step 3. $L_{\min }=(58,13,8 ; 0.7)_{\mathrm{LR}}$.

Step 4. See Table 1.

Step 5. Path $P_{3}$, that is $1-3-5-6$, is the fuzzy shortest path since it has the highest level $\lambda$-triangular LR intersection index, and the corresponding shortest path length is $L_{3}=$ $(58,13,15 ; 0.7)_{\text {LR }}$. 


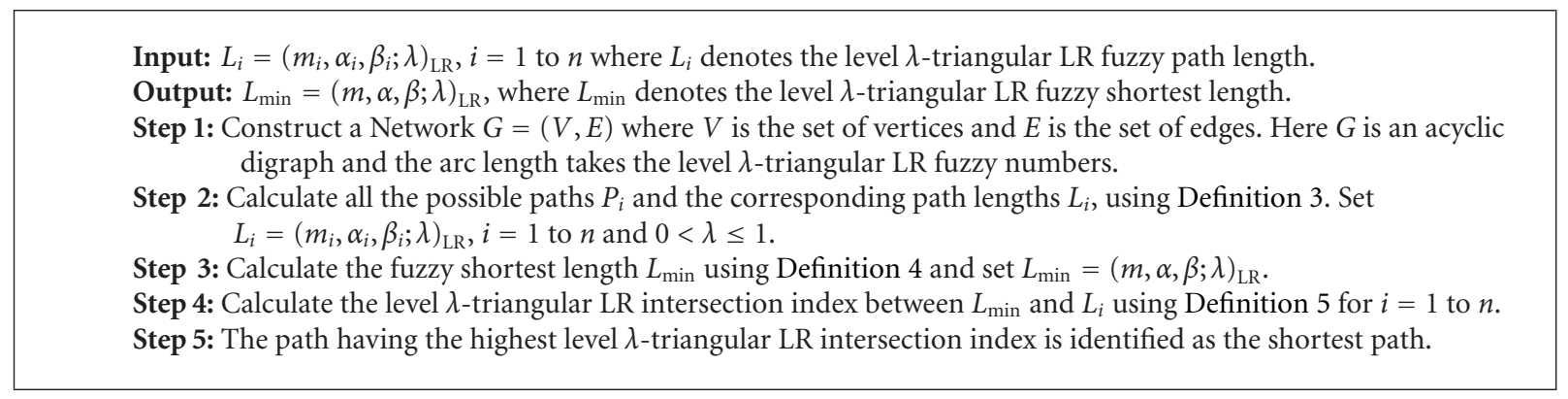

\section{Algorithm 1}

Step 1 and Step 2 are same as in the Algorithm 1.

Step 3: Calculate the level $\lambda$-triangular LR mean and centroid index for each possible path lengths $L_{i}$, using Definition 7.

Step 4: The path having the minimum level $\lambda$-triangular LR mean and centroid index is identified as the shortest path and the corresponding path length is the shortest path length.

Algorithm 2

TABLE 1: Results of the network based on level $\lambda$-triangular LR intersection index.

\begin{tabular}{lcc}
\hline Paths & $T_{\mathrm{LRI}}\left(L_{\min }, L_{i}\right)$ & Ranking \\
\hline$P_{1}: 1-2-4-6$ & 0.11 & 3 \\
$P_{2}: 1-2-5-6$ & 0.54 & 2 \\
$P_{3}: 1-3-5-6$ & 0.7 & 1 \\
\hline
\end{tabular}

TABLE 2: Results of the network based on level $\lambda$-triangular LR weighted average index.

\begin{tabular}{lcc}
\hline Paths & $Z_{i}^{*}\left(L_{\min }, L_{i}\right)$ & Ranking \\
\hline$P_{1}: 1-2-4-6$ & 63.5 & 3 \\
$P_{2}: 1-2-5-6$ & 60 & 2 \\
$P_{3}: 1-3-5-6$ & 58 & 1
\end{tabular}

Path $P_{3}$, that is 1-3-5-6, is the fuzzy shortest path since it has the minimum level $\lambda$-triangular LR weighted average index.

TABLE 3: Results of the network based on level $\lambda$-triangular LR distance index.

\begin{tabular}{lcccc}
\hline Paths & $H D\left(L_{\min }, L_{i}\right)$ & $E D\left(L_{\min }, L_{i}\right)$ & $d\left(L_{\min }, L_{i}\right)$ & Ranking \\
& $w=1$ & $w=2$ & $w=3$ & \\
\hline$P_{1}: 1-2-4-6$ & 43 & 25.5 & 21.82 & 3 \\
$P_{2}: 1-2-5-6$ & 15 & 9 & 7.78 & 2 \\
$P_{3}: 1-3-5-6$ & 7 & 7 & 7 & 1 \\
\hline
\end{tabular}

Path $P_{3}$, that is 1-3-5-6, is the fuzzy shortest path since it has the minimum level $\lambda$-triangular LR distance index.

3.1. Comparing the Results Based on Level $\lambda$-Triangular LR Intersection Index with Few Indices Defined in This Paper. See Tables 2 and 3, and Algorithm 2.

\section{Example 13.}

Steps 1 and 2. They are the same as in Example 12.
TABLE 4: Results of the network based on level $\lambda$-triangular LR mean and centroid index.

\begin{tabular}{lccc}
\hline Paths & $T_{\text {Mean }}\left(L_{i}\right)$ & $T_{\text {Centroid }}\left(L_{i}\right)$ & Ranking \\
\hline$P_{1}: 1-2-4-6$ & 71.5 & 70.67 & 3 \\
$P_{2}: 1-2-5-6$ & 61 & 61.33 & 2 \\
$P_{3}: 1-3-5-6$ & 59 & 58.67 & 1 \\
\hline
\end{tabular}

TABLE 5: Results of the network based on signed distance ranking index.

\begin{tabular}{lcc}
\hline Paths & $d\left(L_{i}, 0\right)$ & Ranking \\
\hline$P_{1}: 1-2-4-6$ & 70.25 & 3 \\
$P_{2}: 1-2-5-6$ & 61.5 & 2 \\
$P_{3}: 1-3-5-6$ & 58.5 & 1 \\
\hline
\end{tabular}

Path $P_{3}$, that is $1-3-5-6$, is the fuzzy shortest path since it has the minimum signed distance ranking index and the corresponding shortest path length is $L_{3}$.

TABLE 6: Results of the network based on acceptability index.

\begin{tabular}{lccc}
\hline Paths & $\begin{array}{c}A\left(L_{\min }<L_{i}\right) \\
\lambda=0.7\end{array}$ & $\begin{array}{c}A\left(L_{\min }<L_{i}\right) \\
\lambda=1\end{array}$ & Ranking \\
\hline$P_{1}: 1-2-4-6$ & 0.59 & 0.85 & 3 \\
$P_{2}: 1-2-5-6$ & 0.16 & 0.22 & 2 \\
$P_{3}: 1-3-5-6$ & 0 & 0 & 1 \\
\hline
\end{tabular}

Path $P_{3}$, that is 1-3-5-6, is the fuzzy shortest path since it has the minimum acceptability index, and the corresponding shortest path length is $L_{3}$.

\section{Step 3. See Table 4.}

Step 4. Path $P_{3}$, that is $1-3-5-6$, is identified as the shortest path, since it has the minimum level $\lambda$-triangular LR mean and centroid index, and the corresponding shortest path length is $L_{3}=(58,13,15 ; 0.7)_{\mathrm{LR}}$. 
3.2. Results and Discussions. One way to verify the solution obtained is to make an exhaustive comparison, now comparing the result obtained in this paper with the existing results to generalize our proposed approach. See Tables 5 and 6.

Hence, we find that the solution obtained for FSPP in this paper coincides with the solution of the existing methods.

\section{Conclusion}

Fuzzy shortest path length and the shortest path are the useful information for the decision makers in a fuzzy shortest path problem. Due to its practical application, many researchers have focused on the fuzzy shortest path problem, and some algorithms were developed for the same. Hence, in this paper, we have defined few indices and developed the new algorithms based on them and verified with the existing methods. The ranking given to the paths is helpful for the decision makers as they make decision in choosing the best of all the possible path alternatives. Hence, we conclude that the algorithms developed in the current research are the simplest and the alternative method for getting the shortest path in fuzzy environment.

\section{Acknowledgment}

The authors are grateful to the referees for their suggestions to improve the presentation of the paper.

\section{References}

[1] L. A. Zadeh, "Fuzzy sets as a basis for a theory of possibility," Fuzzy Sets and Systems, vol. 1, no. 1, pp. 3-28, 1978.

[2] D. Dubois and H. Prade, Fuzzy Sets and Systems: Theory and Applications, Academic Press, New York, NY, USA, 1980.

[3] C. M. Klein, "Fuzzy shortest paths," Fuzzy Sets and Systems, vol. 39, no. 1, pp. 27-41, 1991.

[4] K. C. Lin and M. S. Chern, "The fuzzy shortest path problem and its most vital arcs," Fuzzy Sets and Systems, vol. 58, no. 3, pp. 343-353, 1993.

[5] S. Okada and T. Soper, "A shortest path problem on a network with fuzzy arc lengths," Fuzzy Sets and Systems, vol. 109, no. 1, pp. 129-140, 2000.

[6] T. N. Chuang and J. Y. Kung, "The fuzzy shortest path length and the corresponding shortest path in a network," Computers and Operations Research, vol. 32, no. 6, pp. 1409-1428, 2005.

[7] J. S. Yao and F. T. Lin, "Fuzzy shortest-path network problems with uncertain edge weights," Journal of Information Science and Engineering, vol. 19, no. 2, pp. 329-351, 2003.

[8] S. M. A. Nayeem and M. Pal, "Shortest path problem on a network with imprecise edge weight," Fuzzy Optimization and Decision Making, vol. 4, no. 4, pp. 293-312, 2005.

[9] A. Sengupta and T. K. Pal, "On comparing interval numbers," European Journal of Operational Research, vol. 127, no. 1, pp. 28-43, 2000.

[10] L. Sujatha and R. Sattanathan, "Fuzzy shortest path problem based on triangular LR type fuzzy number using acceptability index," International Journal of Engineering and Technology, vol. 6, pp. 575-578, 2009.

[11] M. Blue, B. Bush, and J. Puckett, "Unified approach to fuzzy graph problems," Fuzzy Sets and Systems, vol. 125, no. 3, pp. 355-368, 2002.
[12] K. P. Sudhir and P. Rimple, Fuzzy Sets and Their Applications, Pragati Prakashan, Meerut, India, 1st edition, 2006.

[13] M. I. Henig, "Efficient interactive methods for a class of multiattribute shortest path problems," Management Science, vol. 40, no. 7, pp. 891-897, 1994.

[14] L. K. Hyung, Y. S. Song, and K. M. Lee, "Similarity measure between fuzzy sets and between elements," Fuzzy Sets and Systems, vol. 62, no. 3, pp. 291-293, 1994.

[15] X. C. Liu, "Entropy, distance measure and similarity measure of fuzzy sets and their relations," Fuzzy Sets and Systems, vol. 52, no. 3, pp. 305-318, 1992.

[16] C. P. Pappis and N. I. Karacapilidis, "A comparative assessment of measures of similarity of fuzzy values," Fuzzy Sets and Systems, vol. 56, no. 2, pp. 171-174, 1993.

[17] J. Ramík and J. Rímánek, "Inequality relation between fuzzy numbers and its use in fuzzy optimization," Fuzzy Sets and Systems, vol. 16, no. 2, pp. 123-138, 1985.

[18] H. Tanaka, H. Ichihashi, and K. Asai, "A formulation of fuzzy linear programming problem based on comparison of fuzzy numbers," Control and Cybernetics, vol. 13, pp. 185-194, 1984.

[19] W. J. Wang, "New similarity measures on fuzzy sets and on elements," Fuzzy Sets and Systems, vol. 85, no. 3, pp. 305-309, 1997. 

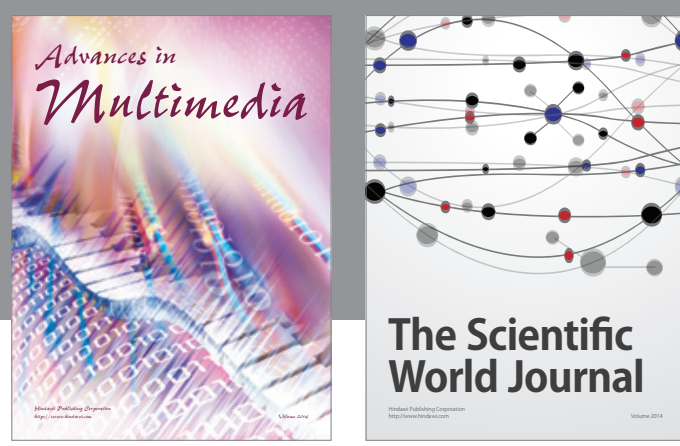

The Scientific World Journal
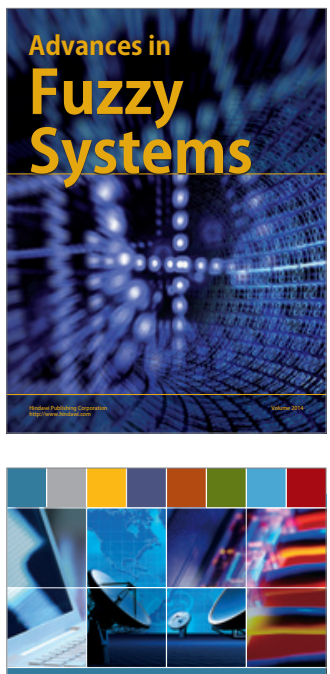

Computer Networks and Communications
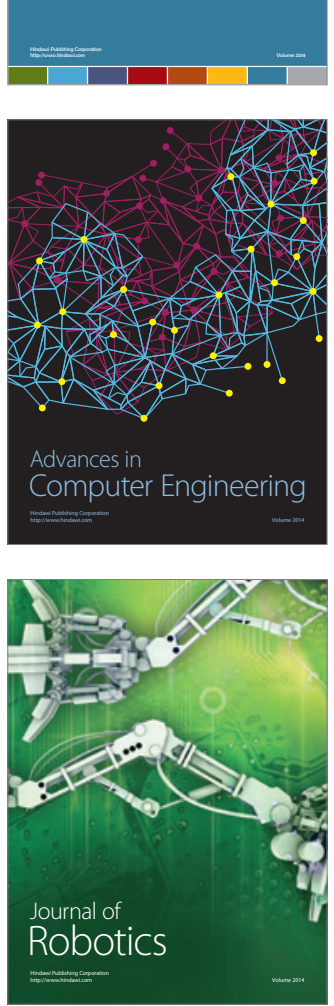
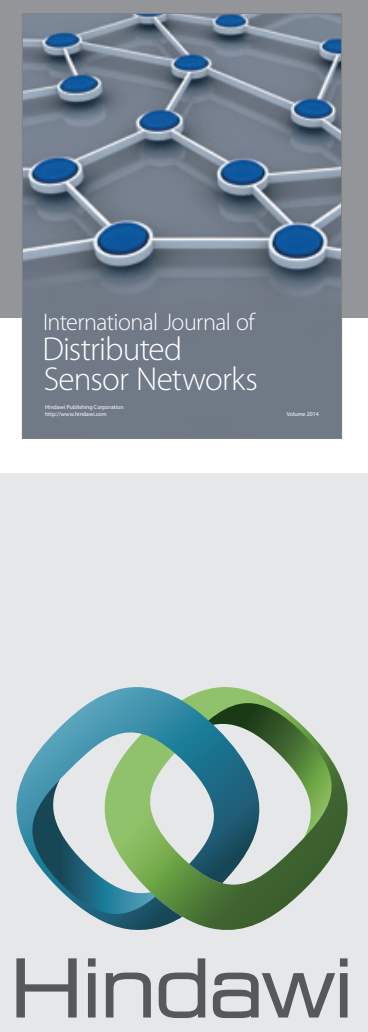

Submit your manuscripts at

http://www.hindawi.com
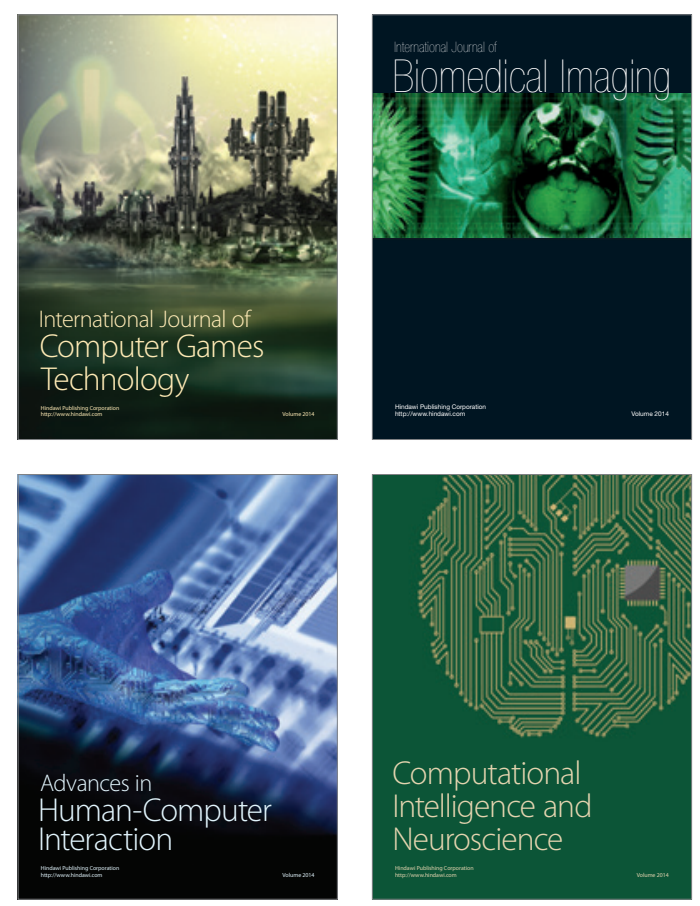
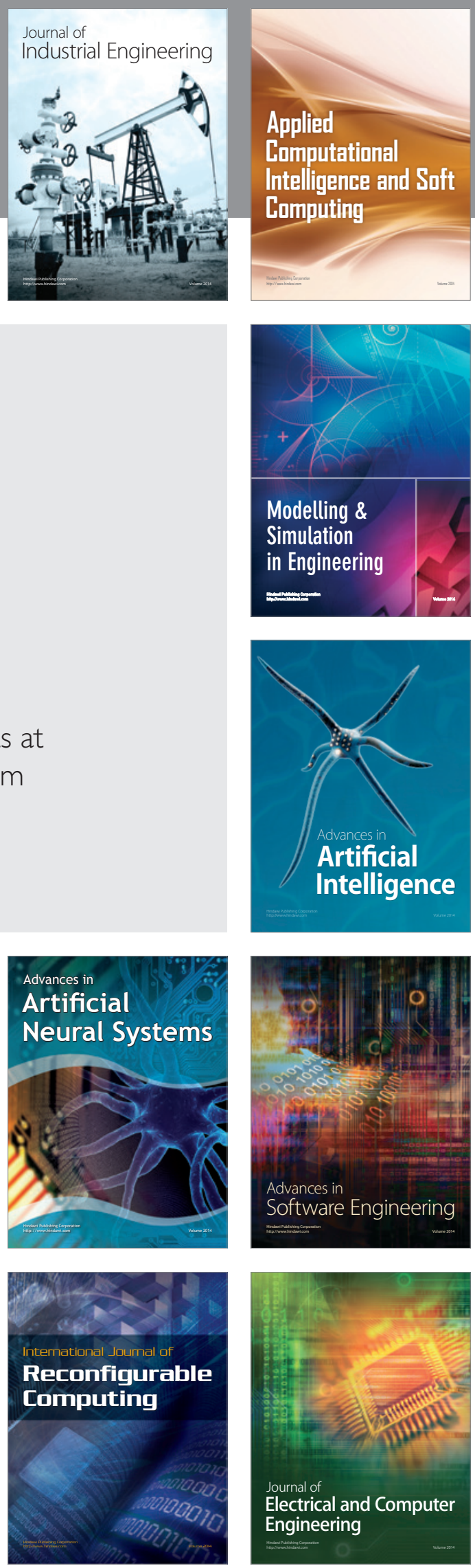